\title{
- Mean Ages at Parities: An Indirect Estimation
}

\author{
MOHAMMAD AFZAL and M. F. K. KIANI
}

\section{INTRODUCTION}

This paper is the outcome of a continuous concern that some of the basic demographic estimates provided by successive surveys in the country do not fulfil the expectation of use at their face value. In a previous paper, an indirect assessment of the birth rates from these surveys was made on the basis of internal consistency of the relevant data. It was pointed out in that paper that a rate being a ratio of the two estimates (e.g., the number of births and the population), any variation of emphasis on obtaining accuracy of the numerator or denominator makes the rate unrealistic. For example, placing more emphasis on a better coverage of births or deaths and a relatively less or no corresponding effort on the measurement of population may lead to biased estimates. Further, any inconsistency of such measurement between different surveys would make their utility for assessing even a time-trend questionable [Afzal et al. (1993)]. Whereas there is no substitute for realistically estimated birth rates (or fertility rates), an index based on relatively simple information which is less affected by the variations in adjustments of births and population is the "Mean Number of Children Ever Born per Woman".

The estimates of mean number of children ever born per woman are based on a simple question about how many children have so far been born alive to a woman till the time of collection of data in a survey or a census. Based on these data, estimates of mean number of children born to women of a specific age group or collectively of all age groups within the reproductive period are easily computed. For a specific age group, these estimates reflect fertility experience since the beginning of the reproductive span, usually taken as 15 years. Thus, for a younger age group of women, the number of children ever born is less than for those in the higher age groups. Collectively for the women aged 15 to 49 years, the estimated mean number of children ever born per woman gives a composite index relating to varied exposure periods. For the purposes of illustrating the approach taken in this paper, the data on children ever born to women as reported by the 1975 Pakistan Fertility Survey (PFS) and the 1990-9.1 Pakistan

Mohammad Afzal is Joint Director and M. F. K. Kiani is Research Demographer at the Pakistan Institute of Development Economics, Islamabad.

Authors' Note: We appreciate the suggestions made by Mohammad Irfan and Mehreen Afzal on an earlier draft of this paper. 
Demographic and Health Survey (PDHS) have been used [Government of Pakistan (1976); NIPS and DHS IRD/Macro (1992)].

Table 1 indicates a slight decline in the average cumulative fertility for the ever married as well as currently married women reported to be still in the reproductive ages (15 to 49) at the time of the 1975 Pakistan Fertility Survey and the.1990-91 Pakistan Demographic and Health Survey. Among the women by age groups, only those who were 15-19 show no such decline. This may possibly be due to an increase in age at marriage over this period, with the first birth occurring a bit sooner than before.'

Table 1

Mean Number of Children Ever Born per Ever Married Woman and per Currently Married Woman: 1975 PFS and 1990-91 PDHS

\begin{tabular}{|c|c|c|c|c|}
\hline \multirow{2}{*}{$\begin{array}{l}\text { Age of } \\
\text { Women }\end{array}$} & \multicolumn{2}{|c|}{ Ever Married Women } & \multicolumn{2}{|c|}{ Currently Married Women } \\
\hline & 1975 PFS & 1990-91 PDHS & 1975 PFS & 1990-91 PDHS \\
\hline $15-19$ & 0.6 & 0.8 & 0.6 & 0.6 \\
\hline $20-24$ & 1.9 & 1.6 & 1.9 & 1.6 \\
\hline $25-29$ & 3.4 & 3.0 & 3.4 & 3.1 \\
\hline $30-34$ & 5.1 & 4.5 & 5.2 & 4.6 \\
\hline $35-39$ & 6.2 & 5.6 & 6.4 & 5.7 \\
\hline $40-44$ & 7.2 & 6.4 & 7.5 & 6.5 \\
\hline $45-49$ & 7.1 & 6.5 & 7.4 & 6.6 \\
\hline All $15-49$ & 4.3 & 4.1 & 4.3 & 4.1 \\
\hline
\end{tabular}

Note: Figures are rounded.

\section{OBJECTIVE}

The estimate of the average number of children ever born provides a measure of cumulative fertility of the sample of women for which data are collected at a specific time. This is also synonymous with the concept of "parity".

The distribution of women by their age at the time of data collection and the ordinal number of the last live birth, i.e., the children ever born, provide a basis for studying their reproductive behaviour. The objective of this paper is to describe an indirect approach for estimating the age patterns of occurrence of births by parity. The main concern here is not of estimating the frequency of occurrence of births (in a period of time) in relation to the population (birth rate) or in relation to the number of females (fertility). Rather, the focus here is on the ages of first, second, and subsequent births,

${ }^{1}$ While estimates of Singulate Mean Age at Marriage provided in Table 2 clearly show an increase over this period, the results of this study regarding mean age at different parities provide a further insight into these phenomenon of women's reproduction. 
and on their shifts over time. Essentially, the approach is an extension of Hajnal's method for using proportions single to estimate singulate mean age at marriage [Hajnal (1954)]. This is an attempt to use relatively simple information on children ever born to get a cross-sectional index that can be used as an indicator of the effects of shifting age at marriage and, subsequently, family formation. These effects may be due to any of the commonly known reasons such as female education and awareness/practice of family planning.

\section{METHODOLOGY}

By using proportions single in each age group (within the ages 15 to 49) of a given sample of women (or men), Hajnal's method gives the average number of years lived by a cohort in a single state by those who marry before age 50 . For estimating singulate mean age at marriage, the proportions single among the females (or males) are applied to a hypothetical cohort (passing through life) to work out the person-years lived in a single state as the cohort passes through each age group before they reach the age of 50 . Taking 15 years as the lowest age where all persons are taken to have lived in a single state, the number of person-years lived by a cohort is $15 \times 1=15$. To this is added the sum of proportions single in each age group $\left(P S_{i}\right)$ multiplied by the number of years of each interval, which is 5 for each of the five-year age groups in the age span 15 to 49 . Out of this, the number of person-years lived by those who did not marry by age 50 are subtracted. The proportion that gets married by age 50 is obtained by averaging percentages ever married reported in the age groups $45-49$ and 50-54. Their estimate can also be obtained by subtracting from the cohort the numbers of those who did not marry by the age of 50 . The total number of the person-years lived in the single state so obtained is divided by the number (or proportion) of persons who ever marry by 50 .

The singulate mean age at marriage based on proportions single in the seven groups of five-year age-span within the age span of 15 to 49 years is estimated as follows. ${ }^{2} S M A M_{15-49}=$

$$
\frac{15+5^{*}\left(P S_{15-19}+P S_{20-24}+\ldots+P S_{45-49}\right)-50 *\left(P S_{45-49}+P S_{50-54}\right) / 2}{1-50 *\left(P S_{45-49}+P S_{50-54}\right) / 2}
$$

This can also be written as:

$$
\frac{30+5 *\left(P S_{15-19}+P S_{20-24}+\ldots+P S_{45-49}\right)-50 *\left(P S_{45-49}+P S_{50-54}\right)}{2-50 *\left(P S_{45-49}+P S_{50-54}\right)}
$$

Following this procedure, the singulate mean age at marriage for females in the

${ }^{2}$ For the mathematical basis of this formula, see Hajnal (1954). 
ages between 15 to 49 , from the 1975 Pakistan Fertility Survey, ${ }^{3}$ comes to 19.6; and from the 1990-91 Pakistan Demographic and Health Survey, to 21.6.

Table 2

Proportions of Women Reported as Single in Each Age Group and Estimates of Singulate Mean Age at Marriage (SMAM): PFS 1975 and PDHS 1990-91

\begin{tabular}{lcccc}
\hline $\begin{array}{l}\text { Age } \\
\text { Group } \\
\text { (i) }\end{array}$ & $\begin{array}{c}\text { Proportion } \\
\text { Single PS }_{(\mathrm{i})}\end{array}$ & SMAM & \multicolumn{2}{c}{ PDHS 1990-91 } \\
\hline $15-19$ & 0.60 & SMAM $_{15-49}=19.6$ & 0.75 & Proportions $^{\text {Single PS }}$ \\
$20-24$ & 0.22 & & 0.39 & \\
$25-29$ & 0.09 & SMAM $_{15-34}=19.3$ & 0.14 & SMAM $_{15-49}=21.6$ \\
$30-34$ & 0.03 & SMAM $_{35}=35.1$ & 0.04 & SMAM $_{35}=35.1$ \\
$35-39$ & 0.02 & & 0.02 & \\
$40-44$ & 0.01 & & 0.02 & \\
$45-49$ & 0.01 & & 0.02 & \\
$50-54$ & 0.01 & & 0.01 & \\
\hline
\end{tabular}

Just as proportions single in age group are used to estimate Singulate Mean Age at Marriage, the basic input for working out the desired estimates of mean age at a parity ' $n$ ' are the age-specific proportions of women in the next lower parity state $(n-1)$.

Following the same argument and approach, this formula has been adopted to work out the mean age of women at first, second, third, fourth, fifth, and sixth parities. For example, the mean age at first parity (birth) may be obtained by estimating the average number of person-years lived in the 'zero birth' state by those ever married or alternately by those who are currently married, over ages 15 to 50 . The single women are to be excluded because fertility in Pakistan is almost entirely associated with married women.

Mean age at second birth is obtained by estimating the average number of person-years lived by the women in one-birth or parity-one state. For working out the mean age at third parity, the average of the person-years lived in parity-two state have to be obtained. Similarly, the average number of person-years lived in parity-three state would yield mean age at fourth parity. In general, for working out the mean age at a specific parity ' $n$ ', the average number of person-years lived in one less or ' $n-1$ ' parity' are needed.

\footnotetext{
${ }^{3}$ For example, in Table 2, the SMAM for 1975 is obtained as follows:

$$
\begin{gathered}
30+5^{*}(0.60+0.22+0.09+0.03+0.02+0.01+0.01)-50(0.01+0.01)=19.6 \\
2-50^{*}(0.01+0.01)
\end{gathered}
$$
}




\section{Distribution of Women by Parity}

The distributions of ever married women and currently married women by parity within each age group as reported by PFS 1975 and PDHS 1990-91 are provided in Table 3. Although the total number of women in each age group is different, their distribution has been made by considering their total as 1 . This has been done to make the comparison by parity easier. For the purposes of this paper, the distributions by the respective proportions for each age group are presented separately up to the fifth parity (or to lower parity if no women of higher parity exist) and the proportions of the sixth and above parities are added up. Consider the women reported to be the age group ' $i$ ' (varying from 15-19 to 45-49), and ' $n$ ' parity (varying from ' 0 ' to ' $6>$ ') as $P^{n}{ }_{i}$. For any age group ' $i$ ' the total of these proportions in an age group is as follows:

$$
P_{i}^{0}+P_{i}^{1}+P_{i}^{2}+P_{i}^{3}+P_{i}^{4}+P_{i}^{5}+P_{i}^{6>}=1 .
$$

Just as Proportions never married (proportion single) are exposed to the chance of getting married, the proportions of only such women who are in the next lower parity $\left(P W^{n-1}{ }_{i}\right)$ are exposed to the risk of giving $n$ th-order birth. Therefore, the second step is to work out the adjusted proportions of women $\left(P W^{n}{ }_{i}\right)$ from proportions $\left(P^{n}{ }_{i}\right.$ given in Table 3). $P W^{n}{ }_{i}$ represents the proportion of women out of the total for each group from which the number of women in the lower than $n-1$ parity/parities are excluded. The total of all women in each age being 1, the estimate of adjusted proportion of women with parity ' $n$ ' in the age group is as follows:

$$
P W_{i}^{n}=P_{i}^{n} \div\left(1-P^{<n-1}\right) \text {. }
$$

The adjusted proportion of women in age group ' $i$ ' with zero parity is as follows:

$$
P W_{i}^{0}=P_{i}^{0} \div\left(1-P^{<0-1}{ }_{i}\right)=P_{i}^{0} \div 1=P_{i}^{0}
$$

Since women of less than zero parity do not exist, the adjusted proportions of women in parity zero for each age are the same as the proportion under parity zero given in Table 3. For example, adjusted proportion for zero parity women, $P W^{0}{ }_{15-19}=$ $P^{0}{ }_{15-19} \div\left(1-P^{<0}{ }_{15-19}\right)=P^{0}{ }_{15-19} \div 1=0.56 \div 1=0.56$. It may be kept in view that $P^{<0}{ }_{15-19}$ $=0$, because no women in parity less than zero exist.

For parity-one women, the adjusted proportions are as follows: ${ }^{4}$

$$
P W_{i}^{1}=P_{i}^{1} \div\left(1-P^{<1-1}\right)=P_{i}^{1} \div\left(1-P_{i}^{0}\right)
$$

\footnotetext{
${ }^{4}$ The adjusted proportions for women with one parity, namely, $P W_{15-19}^{1}=P_{15-19}^{1} \div\left(1-P^{<1-1}{ }_{15-19}\right)$ $=P_{15-19}^{1} \div\left(1-P^{<1}{ }_{15-19}\right)=0.32 \div(1-0.56)=0.73$. Similarly, the adjusted proportion for parity -2 women in the same age group is as follows: $P W^{2}{ }_{15-19}=0.11 \div[1-(0.56+0.32)]=0.92$.
} 


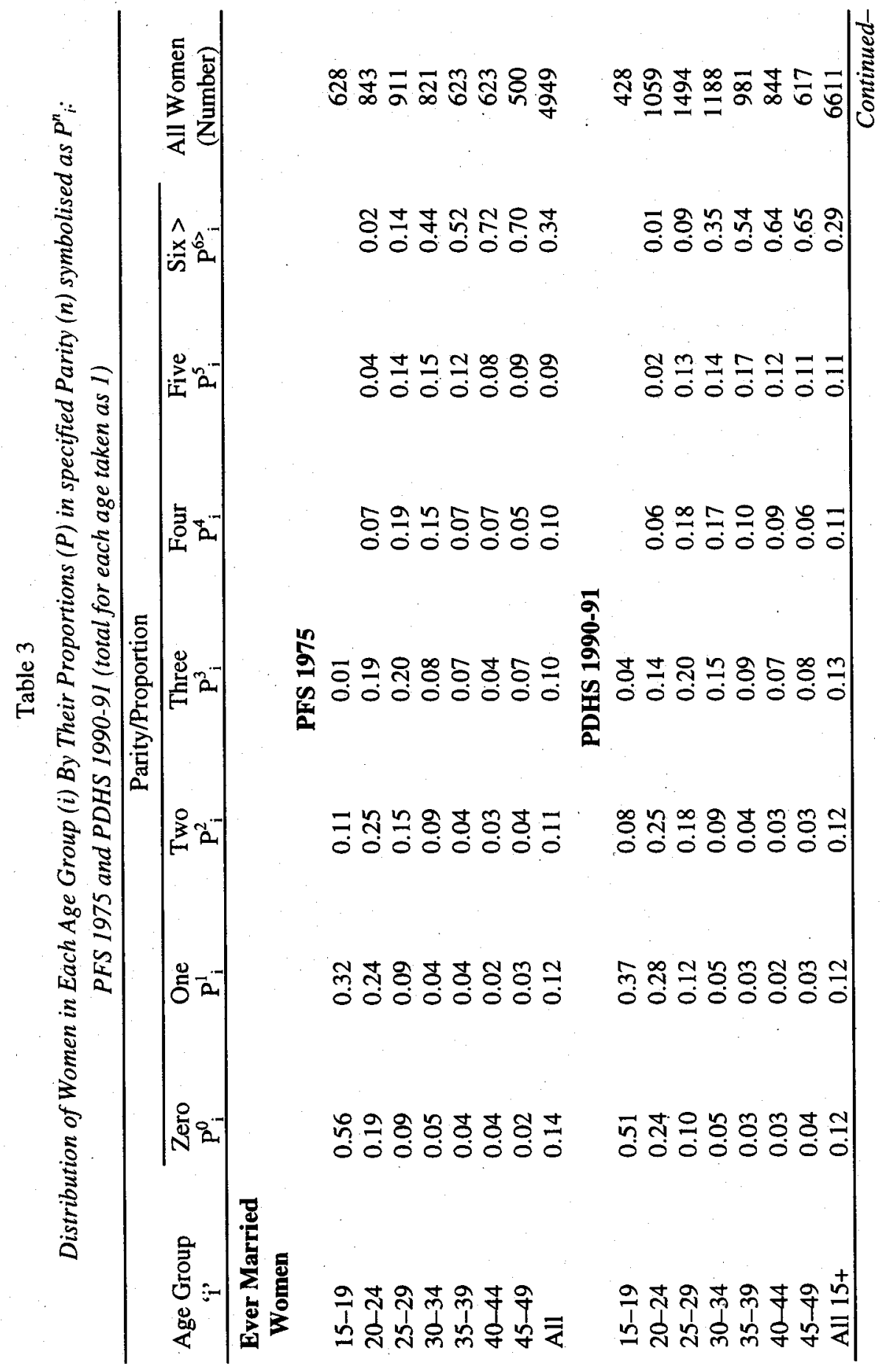




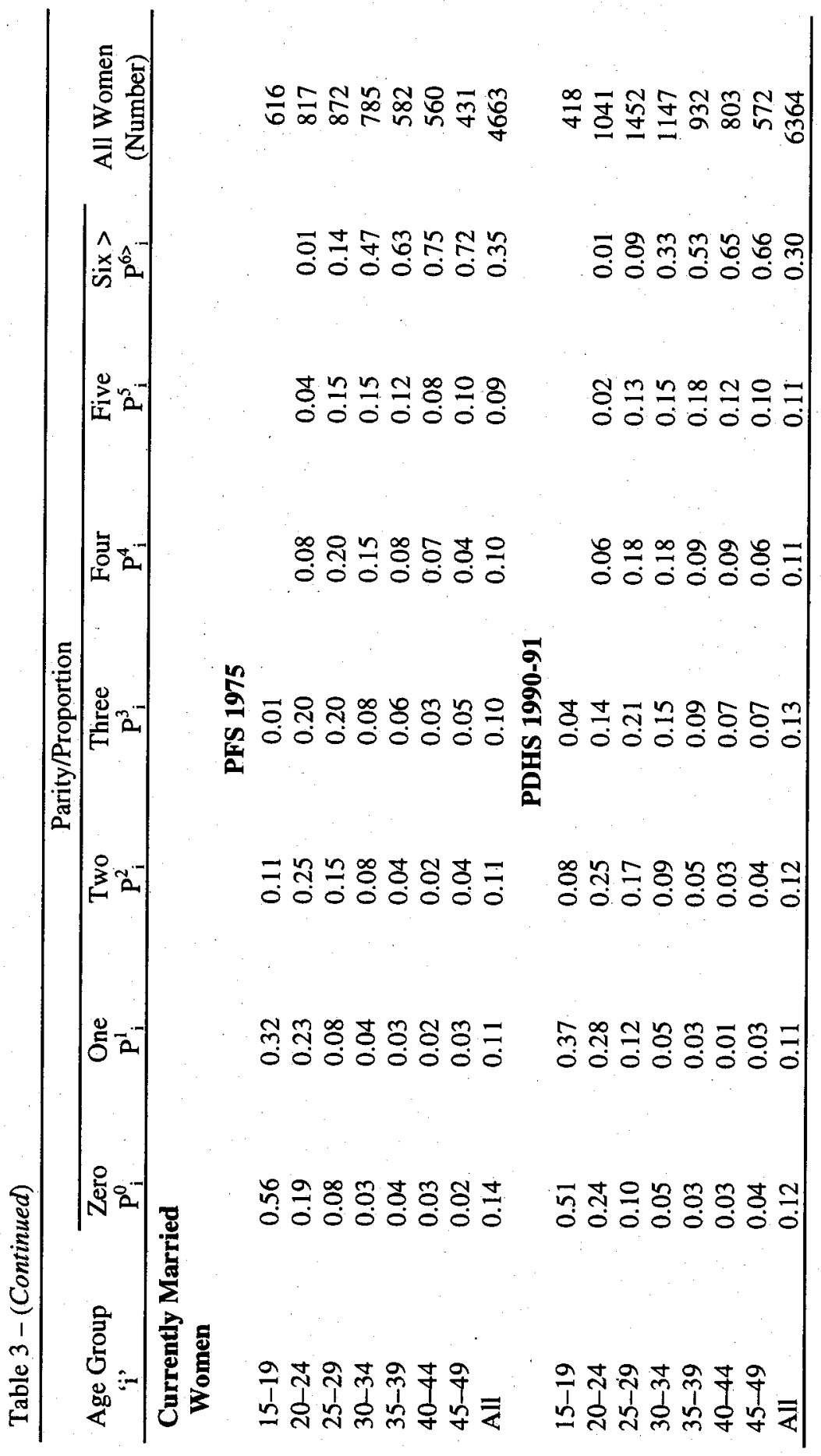


For higher parities, the adjusted proportions are as follows:

Parity $2 P W_{i}^{2}=P_{i}^{2} \div\left[1-\left(P_{i}^{1}+P_{i}^{0}\right)\right.$

Parity $3 P W_{i}^{3}=P_{i}^{3} \div\left[1-\left(P_{i}^{2}+P^{1}{ }_{i}+P_{i}^{0}\right)\right.$

Parity $4 P W_{i}^{4}=P_{i}^{4}+\left[1-\left(P_{i}^{3}+P_{i}^{2}+P^{1}{ }_{i}+P^{0}{ }_{i}\right)\right.$

Parity $5 P W_{i}^{5}=P_{i}^{5} \div\left[1-\left(P^{4}{ }_{i}+P^{3}{ }_{i}+P^{2}{ }_{i}+P^{1}{ }_{i}+P_{i}^{0}\right)\right.$

The estimates so obtained for ever married and currently married women are described in Table 4.

\section{MEAN AGES AT PARITIES}

The third step involves the estimation of the mean age of women at different parities $\left(M W^{n}{ }_{15-49}\right)$ from adjusted proportions $P W^{n}$, given in Table 4 . Among the women reported to be the age group 15-19, the adjusted proportion of those with 'zero' is $P W_{15-19}^{0}$.

The corresponding symbols for the adjusted proportions of women in the remaining seven age groups are $P W_{20-24}^{0}, P W_{25-29}^{0}, P W_{30-34}^{0}, P W_{35-39}^{0}, P W_{40-44}^{0}$, and $P W^{0}{ }_{45-49}$. Since the values $P W_{50-54}^{0}$ were not available, these are taken to be the same as $P W_{45-49}^{0}$, because there is a very small difference between the adjusted proportions of even the previous two age groups. The estimate of the average number of person-years completed, or mean age at first parity for women over the age span 15 to 49 , would be as follows: ${ }^{5} M W_{15-49}^{1}=$

$$
\frac{30+5 *\left(P W_{15-19}^{0}+P W_{20-24}^{0}+\ldots+P W_{45-49}^{0}\right)-50 *\left(P W_{45-49}^{0}+P W_{50-54}^{0}\right)}{2-50 *\left(P W_{45-49}^{0}+P W_{50-54}^{0}\right)}
$$

Following the same formula, the adjusted proportions for women in zero parity in the ages between 15 to 34 (or less than 35 ) have been used to estimate the mean age at first birth occurring before age 35 . Similarly, by using the adjusted proportion for women of zero parity in the ages 35 years and above, who remained without a birth before reaching age 35 , the mean age at their first birth occurring from 35 years onwards (till age 50 ) has been provided separately. ${ }^{6}$

${ }^{5}$ For example, by using the adjusted proportions of ever married women from PFS 1975, the estimated mean age at first parity, $M W^{1}{ }_{15-49=}=$

$$
\begin{gathered}
30+5^{*}(0.56+0.19+0.09+0.05+0.04+0.04+0.02)-50(0.02+0.02) \\
2-50^{*}(0.02+0.02)
\end{gathered}=19.34
$$

${ }^{6}$ Corresponding to the mean ages at first parity before age 35 and above 35 , the estimates of Singulate Mean Age at Marriage of women during these two smaller age spans have also been provided in Table 2. 


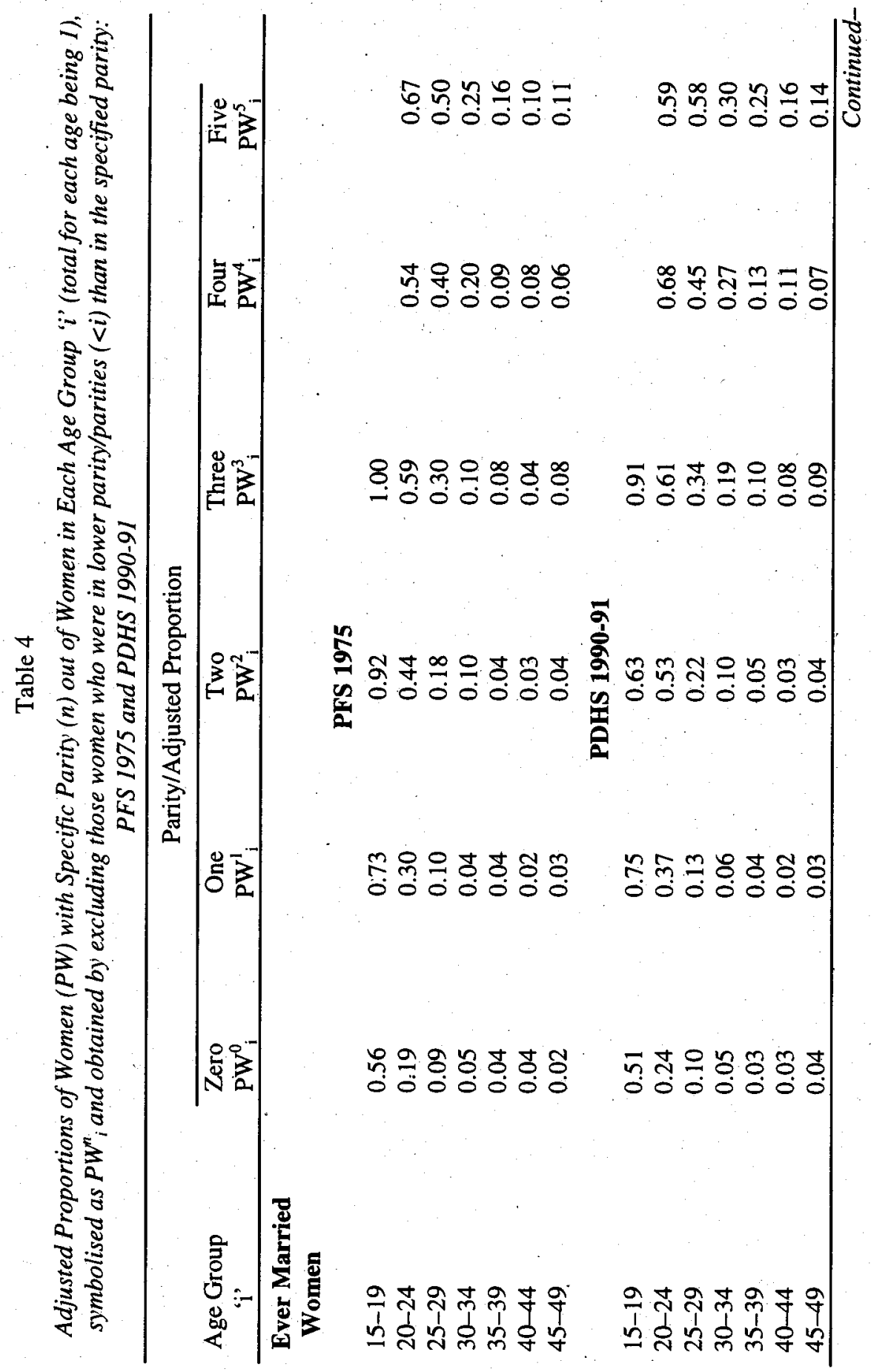




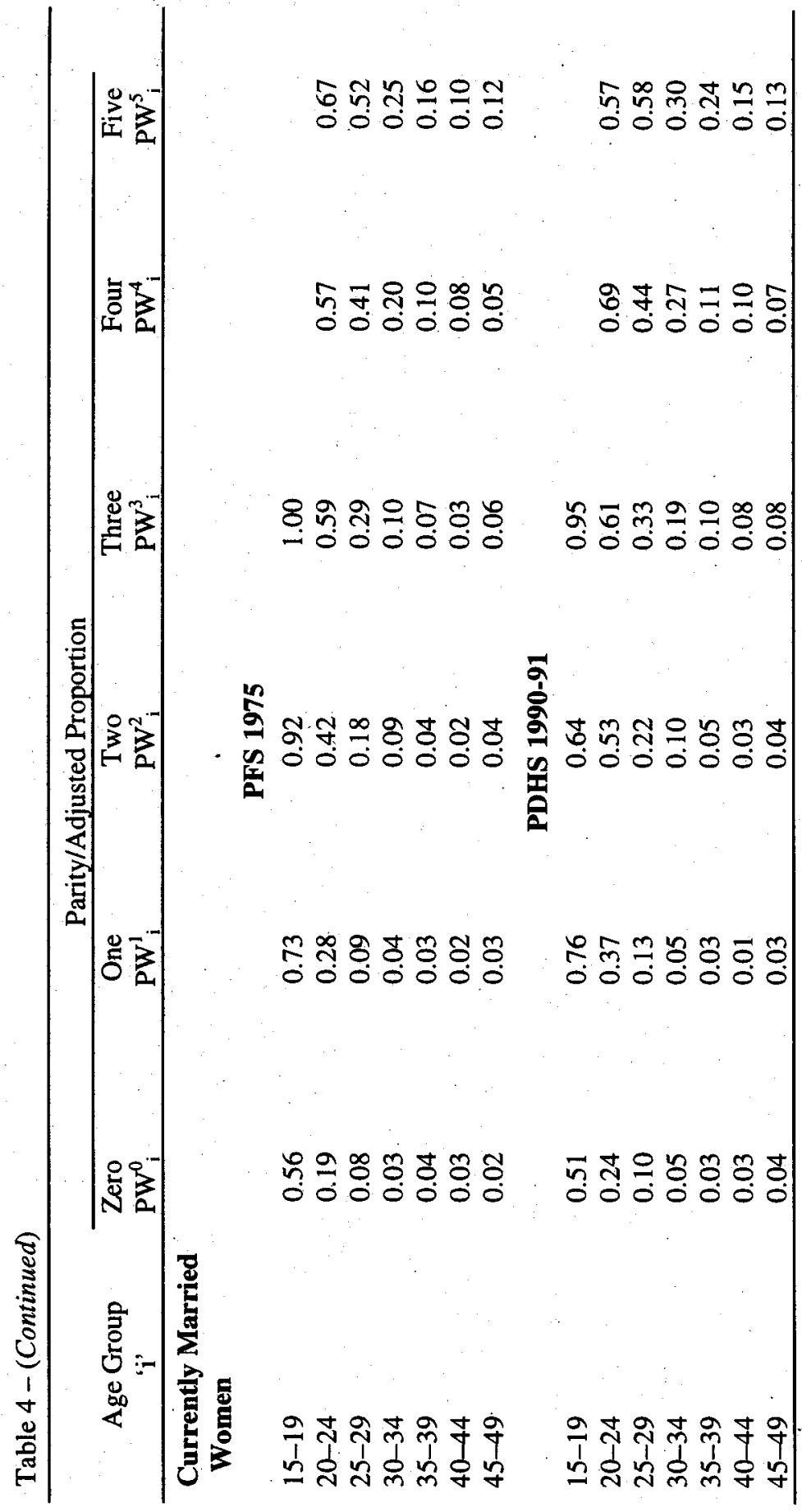


The estimate of mean age at first birth for women in ages less than 35 is as follows: $M W^{15-34}=$

$$
\frac{30+5^{*}\left(P W_{15-19}^{0}+P W_{20-24}^{0}+P W_{25-29}^{0}+P W_{30-34}^{0}\right)-35 *\left(P W_{30-34}^{0}+P W_{35-39}^{0}\right)}{2-35 *\left(P W_{30-34}^{0}+P W_{35-39}^{0}\right)}
$$

The estimate for ages 35 to $49,\left(M W_{35>}^{1}\right)$ is calculated as

$$
\frac{70+5^{*}\left(P W_{35-39}^{0}+P W_{40-44}^{0}+P W_{45-49}^{0}\right)-50 *\left(P W_{45-49}^{0}+P W_{50-54}^{0}\right)}{2-50 *\left(P W_{45-49}^{0}+P W_{50-54}^{0}\right)}
$$

For estimating the mean age at second parity, the women having no birth, i.e., of zero parity, are excluded, since only those with one parity have the chance of giving a second birth. The mean age at second parity (birth) is obtained by estimating the average number of person-years lived by women of one parity in the 'one birth' state between ages 15 to 50 or over shorter periods as described earlier.

For those in ages 15-19, the adjusted proportion of women with 'one' birth only is mentioned as $P W^{1}{ }_{15-19}$. The corresponding symbols for the adjusted proportions of women in the remaining seven age groups would be $P W_{20-24}^{1}, P W_{25-29}^{1}, P W_{30-34}^{1}, P W_{35-39}^{1}$, $P W_{40-44}^{1}, P W_{45-49}^{1}$ and $P W_{50-54}^{1}$. Following the same approach (as for singulate mean age at marriage), the estimate of mean age at second parity for women over the age span of 15 to $49,\left(M W^{2}{ }_{15-49}\right)$, would be as follows:

$$
\frac{30+5^{*}\left(P W_{15-19}^{1}+P W^{1}{ }_{20-24}+\ldots+P W_{45-49}^{1}\right)-50 *\left(P W^{1}{ }_{45-49}+P W^{1}{ }_{50-54}\right)}{2-50 *\left(P W^{1}{ }_{45-49}+P W^{1}{ }_{50-54}\right)}
$$

The estimate of mean age at second birth for women in ages less than 35 , $\left(M W^{2}{ }_{15-34}\right)$, is as follows:

$$
\frac{30+5^{*}\left(P W^{1}{ }_{15-19}+P W^{1}{ }_{20-24}+P W_{25-29}^{1}+P W^{1}{ }_{30-34}\right)-35^{*}\left(P W_{30-34}^{1}+P W_{35-39}^{1}\right)}{2-35^{*}\left(P W_{30-34}^{1}+P W_{35-39}^{1}\right)}
$$

The estimate for ages 35 to $49,\left(M W_{35>}^{2}\right)$, is calculated as:

$$
\frac{70+5 *\left(P W_{35-39}^{1}+P W_{40-44}^{1}+P W_{45-49}^{1}\right)-50^{*}\left(P W_{45-49}^{1}+P W_{50-54}^{1}\right)}{2-50 *\left(P W_{45-49}^{1}+P W_{50-54}^{\prime}\right)}
$$

For estimating the mean age at third parity, the women having no birth as well as those with one birth are excluded, since only those with a two-parity have the chance of giving a third birth. The mean age at third parity is obtained by estimating the average number of person-years lived by women with two births in the 'two births' state between the ages 15 to 50 or over shorter periods as described earlier. 

$\left(M W^{3}{ }_{15-49}\right)=$

Mean age at third parity for women in ages 15 to 49 would be as follows:

$$
\frac{30+5 *\left(P W_{15-19}^{2}+P W^{2}{ }_{20-24}+\ldots+P W_{45-49}^{2}\right)-50 *\left(P W_{45-49}^{2}+P W^{2}{ }_{50-54}\right)}{2-50 *\left(P W^{2}{ }_{45-49}+P W^{2}{ }_{50-54}\right)}
$$

The estimate of mean age at third birth for women in ages less than $\mathbf{3 5}$ is as follows: $\left(M W^{3}{ }_{15-34}\right)=$

$$
\frac{30+5 *\left(P W_{15-19}^{2}+P W^{2}{ }_{20-24}+P W^{2}{ }_{25-29}+P W^{2}{ }_{30-34}\right)-35 *\left(P W_{30-34}^{2}+P W^{2}{ }_{35-39}\right)}{2-35^{*}\left(P W^{2}{ }_{30-34}+P W^{2}{ }_{35-39}\right)}
$$

The estimate for ages 35 to $49,\left(M W_{35>}^{3}\right)$, is calculated as:

$$
\frac{70+5 *\left(P W^{2}{ }_{35-39}+P W_{40-44}^{2}+P W_{45-49}^{2}\right)-50 *\left(P W_{45-49}^{2}+P W^{2}{ }_{50-54}\right)}{2-50 *\left(P W_{45-49}^{2}+P W^{2}{ }_{50-54}\right)}
$$

The corresponding estimates for mean ages of women at fourth, fifth, and sixth - parity are obtained following similar approaches. However, for the fifth and sixth parity estimates, the formula has to be adjusted for the lowest age group, which in their case is 20-24 instead of 15-19. This is because till the time of data collection no woman in the age group was reported to have given birth to more than three children. For example, the mean age at fourth parity for women in ages 20 to 49 is as follows:

$\left(M W_{20-49}^{4}\right)=$

$$
\frac{40+5^{*}\left(P W_{20-24}^{3}+P W^{3}{ }_{25-29}+\ldots+P W_{45-49}^{3}\right)-50 *\left(P W^{3}{ }_{45-49}+P W^{3}{ }_{50-54}\right)}{2-50 *\left(P W^{3}{ }_{45-49}+P W^{3}{ }_{50-54}\right)}
$$

The estimate of mean age at fourth birth for women in ages less than 35 is as follows:

$$
\begin{aligned}
& \left(M W^{4}{ }_{20-34}\right)= \\
& \quad \frac{40+5^{*}\left(P W_{20-24}^{3}+P W_{25-29}^{3}+P S W_{30-34}^{3}\right)-35^{*}\left(P W_{30-34}^{3}+P W_{35-39}^{3}\right)}{2-35^{*}\left(P W^{3}{ }_{30-34}+P W^{3}{ }_{35-39}\right)}
\end{aligned}
$$

The estimate for ages 35 to $49,\left(M W^{4}{ }_{35>}\right)$, is calculated as:

$$
\frac{70+5 *\left(P W_{35-39}^{3}+P W_{40-44}^{3}+P W_{45-49}^{3}\right)-50 *\left(P W_{45-49}^{3}+P W_{50-54}^{3}\right)}{2-50 *\left(P W_{45-49}^{3}+P W_{50-54}^{3}\right)}
$$


The estimates of mean ages at different parities for ever married women and for currently married women, obtained by following the procedures described above, are given in Table 5.

The table shows that for all ages the estimates of mean age at each parity, except the first, increased between 1975 and 1990-91. Also, the estimated mean age at first parity for both years is lower than the corresponding Singulate Mean Age at Marriage. The apparent decline in age at first parity simply implies that for those who got married within their reproductive age span, the average person-years spent by them in the state of zero birth declined. One main reason behind this was that the singulate mean age at marriage increased over this period from 19.6 to 21.6 . In other words, the period between marriage and first birth was lower for women who were in the reproductive ages in 1990-91, and had spent more years before marriage. It may be mentioned again that only ever married (or currently married) women were included for the estimation of mean ages at parities. In other words, the proportion of unmarried women in age groups which form the basis to compute Singulate Mean Age at Marriage have not been taken into account for this purpose. If these women (having obviously given no birth) are added to those who had married but were still in zero parity state, and if the proportions in zero birth state are worked out accordingly, then the mean age at first parity becomes higher (from 19.34 to 22.1 for 1975 and 18.9 to 23 for $1990-91$ ), ${ }^{7}$ showing an increase (instead of decline) of about a year over the period between the two surveys. ${ }^{8}$

For women in all ages together, the estimated mean age at each parity increased over the period between the two surveys. The slightly lower estimates for the currently married are due to the exclusion of the person-years spent in each parity by those who were widowed, divorced, or separated.

For ever married women, there was an increase in the mean ages at the first two parities in the age span of 15 to 34 , but from age 35 and above, slight declines are indicated. For the third and fourth parities, the changes for the younger span are in the negative, but over the older age span these are positive. For the fifth parity, the increase

${ }^{7}$ The 1990-91 PDHS report provides the estimate of median age at first birth for the ages 25-29 as 21.3. When seen according to the current age of ever married women, the median age goes up from 21 for age group 25-29 and to 22.6 for age group 45-49.

${ }^{8}$ If one considers 22.1 for PFS 1975 and 23 for 1990-91 PDHS as the respective ages at first parity of ever married women, then with differences in the ages at successive parities the same as those indicated from the estimates given in Table 5, the ages at different parities would be as follows:

\begin{tabular}{lllllll}
\hline & & \multicolumn{5}{c}{ Parity } \\
\hline & One & Two & Three & Four & Five & Six \\
\hline $\begin{array}{l}\text { Age at Parity } \\
\text { 1975 PFS }\end{array}$ & 22.1 & 23.1 & 23.4 & 26.6 & 28.1 & 29.1 \\
1990-91 PDHS & 23.0 & 25.3 & 27.3 & 29.0 & 29.1 & 29.1 \\
\hline
\end{tabular}




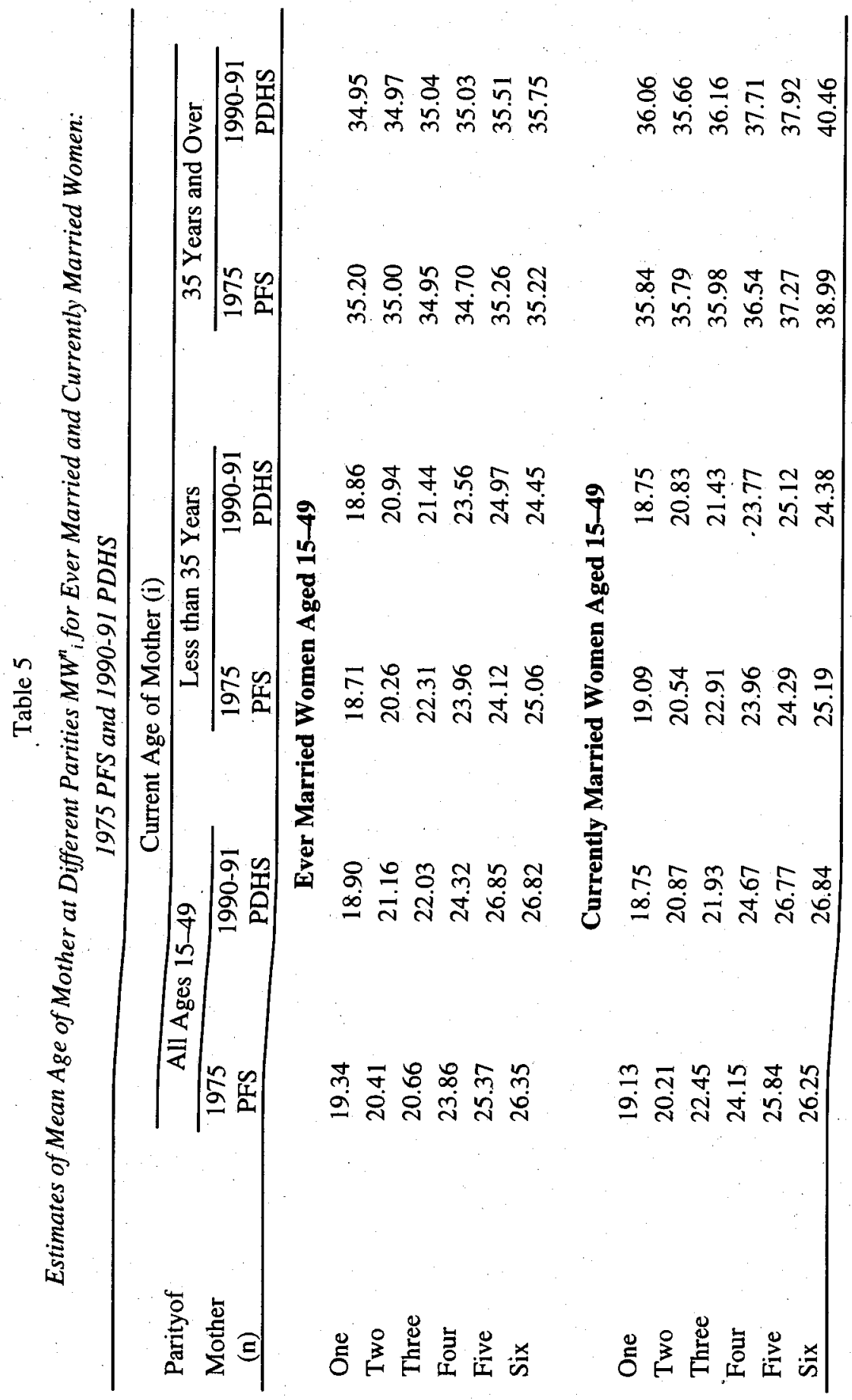


in mean age is indicated for both the age spans; but for the sixth parity the changes are again in the opposite direction. While the effects of inconsistencies in the direction of changes for the two age spans are absorbed in the estimates for the overall age span of 15 to 49 , the estimates for the smaller age spans give some idea of the experiences of different age cohorts. One cannot, however, ignore the small size of the samples in different age groups, which may be a factor contributing to the inconsistencies in the observed changes. Despite these possible reasons, the utility of the indirect approach used for estimating mean ages at different parities from a relatively simple set of data on children ever born seems obvious. If tabulations of ever married or currently married women are made available from large data sets, like the population census or large scale surveys, one can easily utilise these to bring out important estimates relating to the ages of reproduction by order of birth and their changes over time. Since the age at which a woman gives birth for the first time, or for that matter subsequently, has important implications for the family size and population growth, this approach, by not requiring more detailed and complex fertility history data, has some obvious advantages. Furthermore, the results obtained by using this approach are relevant not only in examining the role of family planning programmes but also in assessing the changes due to women's involvement in educational and economic activities.

\section{REFERENCES}

Afzal, Mohammad, M. F. K. Kiani and Ali Mohammad (1993) An Indirect View of Fertility Changes in Pakistan. The Pakistan Development Review 32:4 10811093.

Hajnal, John (1954) Age at Marriage and Proportions Marrying. Population Studies 7: 111-136.

NIPS and DHS IRD/Macro (1992) Pakistan Demographic and Health Survey 19901991. Islamabad: National Institute of Population Studies and Columbia, Maryland: IRD/Macro International, Inc.

Pakistan, Government of (1976) Pakistan Fertility Survey: First Report. Islamabad: Population Planning Council. 


\section{Comments}

The authors have followed a procedure of estimating mean age at first marriage by indirect method, as developed by British demographer John Hajnal and published in Population Studies in 1953. This procedure has been used often since then. It is different from the mean age or the median age at marriage which are directly estimated. It is based on the mean number of years lived by a cohort of women as never married or single.

The total number of those who did not marry before age 50 is divided by the number of those who married. The singulate mean age at marriage thus computed can be used for intercensal inter-surveys or for inter-country comparison. It is different from other procedures as it takes into account both the age at marriage and the proportions married. The authors of this paper have made an attempt to apply this procedure to estimating mean ages at first parity, second parity, and so on.

There are procedures for computing age-specific birth rates by median or mean age of mother based on registration or survey data. Another simple procedure is to classify the births by order of birth or calculate order-specific birth rates by specific age groups of women. In applying the Hajnal procedure, the authors have used the 1974-75 Pakistan Fertility Survey and the 1990-91 Pakistan Demographic and Health Survey data. The extent to which the two surveys varied in terms of contents, field work, and quality, although difficult to determine, would affect the comparison undertaken in the paper. They have reproduced Table 1 from these surveys on the number of children ever born per ever married women and currently married women, but it would have been preferable if the table on the number of children ever born by the age group of total women was also produced. On the basis of their Table 1, the authors argue that "only those who were 15-19 show no decline". They attribute this to "an increase in age at marriage". This seems to be a far-fetched conclusion as a look at the fertility of married women in age group 15-19 shows that there is no difference between the fertility of currently married women of PFS and of those in the corresponding age group of PDHS. Table 4 is labelled as "adjusted proportions of women", which implies that the proportions are not original. Instead the table should have indicated "proportions excluding $n-1, n-2$ parities", etc.

The authors' initiative in extending the existing procedure for estimating mean ages of mothers at different parities is appreciated. But there is a need for further work in this direction. The authors point out that "estimates of mean age at each parity except the first one increased between 1974-75 to 1990-91". And they contend that the mean age at first parity for both years is lower than the corresponding singulate mean age at marriage. It appears that the former indicators are for the first parity only, whereas the latter (singulate mean age) is for all women. It would be more correct if an indicator for all parities was also computed by equating zero parity women, like single women in the Hajnal equation, for calculating singulate mean age at marriage. 
It may be suggested that the authors, focusing on the utility of the exercise, should make a comparison with other existing techniques and show its importance for future use. Of course, some differences could be due to the difference in the quality of data between the two surveys, but the authors' effort is appreciated for initiating work based on logical thinking.

National Institute of Population Studies,

Sultan S. Hashmi Islamabad. 